\title{
Manejo da lagarta-do-cartucho em milho com formulações de nim e Bacillus thuringiensis subsp. aizawai
}

\author{
Management of fall armyworm in corn with commercial neem and Bacillus thuringiensis \\ subsp. aizawai
}

\author{
Marcileyne Pessôa Leite de Lima ${ }^{\mathrm{I}^{*}}$ José Vargas de Oliveira $^{\mathrm{I}}$ \\ Edmilson Jacinto Marques ${ }^{I}$
}

\begin{abstract}
- NOTA-
RESUMO

A lagarta-do-cartucho, Spodoptera frugiperda, principal praga do milho, é comumente controlada com inseticidas sintéticos. Assim, na busca de alternativas eficientes $e$ de baixo impacto ambiental, testou-se em campo as formulações comerciais de nim Natuneem ${ }^{\circledR}\left(10 \mathrm{~mL} \mathrm{~L}^{-1} \mathrm{em} 2005\right.$ e $15 \mathrm{~mL} \mathrm{~L} L^{-1}$ em 2006) e Neemseto ${ }^{\circledR}\left(5\right.$ e $10 \mathrm{~mL} \mathrm{~L} L^{-1}$ em 2005, e 10 e $15 \mathrm{~mL} \mathrm{~L}^{-1}$ em 2006) e do inseticida biológico Bta (Xentari ${ }^{\circledR}$ $10 \mathrm{~g} \mathrm{~L}^{-1}$ em 2005 e 2006), aplicados aos 15,30 e 45 e aos 15 , 25 e 35 dias, após a emergência das plantas, em 2005 e 2006, respectivamente. Antes de cada pulverização foram atribuídas notas de 0 a 5 para as injúrias foliares. Em 2005, somente Neemseto ${ }^{\circledR} 10 \mathrm{~mL} \mathrm{~L} \mathrm{~L}^{-1}$ foi eficiente aos 30 dias. Em 2006,

2005, and 10 and $15 \mathrm{~mL} \mathrm{~L}^{-1}$ in 2006) and biological insecticide Bta Xentari ${ }^{\circledR}\left(10 \mathrm{~g} \mathrm{~L}^{-1}\right.$ in 2005 and 2006) applied at 15, 30 and 45 and at 15, 25 and 35 days after plant emerge in 2005 and 2006, respectively, were tested. Evaluations consisted of using a scale of foliar injury from 0 to $5 r$ before each spray. In 2005, only Neemseto ${ }^{\circledast} 10 \mathrm{~mL} \mathrm{~L}^{-1}$ was efficient at 30 days. In 2006, Neemseto ${ }^{\circledR} 15 \mathrm{~mL} \mathrm{~L}^{-1}$ and Bta Xentari ${ }^{\circledR}$ with average score of 0.93 and 0.98 , respectively, were different from control with score of 2.38 at 35 days. At greenhouse corn plants were treated with Natuneem ${ }^{\circledast}$, Neemseto ${ }^{\circledR}$ and Xentari ${ }^{\circledR}$ insecticides followed by artificial infestation with newly-hatched larvae. After 10 days, it was verified that treatment were equally efficient, differing from the control, regarding to the injury score, as well as, the number of surviving worms.
\end{abstract} Neemseto $^{\circledR} 15 \mathrm{~mL} \mathrm{~L}^{-1}$ e Bta $10 \mathrm{~g} \mathrm{~L}^{-1}$, com notas médias de 0,93e 0,98 , diferiram da testemunha, com nota 2,38, na avaliação realizada aos 35 dias. Em casa-de-vegetação, plantas de milho foram pulverizadas com os inseticidas Natuneem ${ }^{\circledR}$, Neemseto ${ }^{\circledR}$ e Xentari ${ }^{\circledast}$ e posteriormente infestadas, artificialmente, com lagartas recém-eclodidas. Após 10 dias, verificou-se que os tratamentos foram igualmente eficientes, diferindo da testemunha, tanto em relação às notas, quando ao número de lagartas sobreviventes.

Palavras-chave: Meliaceae, Spodoptera frugiperda (J.E. SMITH) (LEPIDOPTERA: NOCTUIDAE), inseticida botânico, bioinseticida, MIP.

\section{ABSTRACT}

The fall armyworm (Spodoptera frugiperda) is the main pest of corn in Brazil and the use of synthetic insecticides is the most commonly applied method to its control. Thus, searching for efficient and low environment impact alternatives, commercial formulations of the neem Natuneem ${ }^{\circledR}\left(10 \mathrm{~mL} \mathrm{~L} \mathrm{~L}^{-1}\right.$ in 2005 and $15 \mathrm{~mL} \mathrm{~L}^{-1}$ in 2006) and Neemseto ${ }^{\circledast}\left(5\right.$ and $10 \mathrm{~mL} \mathrm{~L}^{-1}$ in
Key words: Meliaceae, fall armyworm, botanical insecticide, bioinsecticide, MIP.

A cultura do milho, Zea mays L., é de grande relevância na alimentação humana, na animal e como matéria-prima na indústria de alimentos. Nas regiões de sequeiro do Nordeste, o milho assume caráter de cultura de subsistência e, portanto, a redução da produção afeta diretamente a disponibilidade de alimento para a população de baixa renda. O potencial produtivo do milho pode ser afetado, principalmente, pela competição com plantas daninhas nos estágios iniciais de desenvolvimento e o ataque de pragas, sobretudo a lagarta-do-cartucho, Spodoptera frugiperda (J.E. Smith, 1797) (Lepidoptera: Noctuidae), que pode reduzir a produção de grãos em até $34 \%$ (CRUZ, 1995).

'Departamento de Agronomia - Entomologia, Universidade Federal Rural de Pernambuco (UFRPE), 52171-900, Recife, PE, Brasil. E-mail: marcileynepessoa@hotmail.com. *Autor para correspondência. 
Os inseticidas sintéticos são utilizados como tática preferencial no controle de $\boldsymbol{S}$. frugiperda, em milho no Brasil, sem levar em consideração os princípios do manejo ecológico de pragas, contribuindo para a elevação dos custos de produção e dos riscos ambientais decorrentes do uso inadequado desses produtos (CRUZ, 1995). Por outro lado, inseticidas à base de nim e biológicos, à base da bactéria Bacillus thuringiensis Berliner (Bt), devido a sua eficiência, baixa toxicidade para o homem e animais e também para diversos inimigos naturais de pragas, são de grande importância para serem avaliados, em programas de manejo integrado de $\boldsymbol{S}$. frugiperda, em cultivos de milho tradicional e orgânico (MARTINEZ \& VAN EMDEN, 2001; MARTINEZ, 2002; SIEGEL, 2001).

Diante das considerações apresentadas, objetivou-se, no presente trabalho obter informações mais precisas sobre o uso de formulações comerciais de nim e de inseticida biológico, no controle de $\boldsymbol{S}$. frugiperda, em casa-de-vegetação e campo.

\section{Experimento de campo - safra 2005}

No primeiro semestre de 2005, instalou-se experimento no Sítio do Araçá, Km13, em Camaragibe (Aldeia), PE, com o milho híbrido duplo AG 405. Utilizaram-se as formulações comerciais de nim (Natuneem $^{\circledR} 10 \mathrm{~mL} \mathrm{~L}^{-1}$, Neemseto ${ }^{\circledR} 5$ e $10 \mathrm{~mL} \mathrm{~L}^{-1}$ ) e $\boldsymbol{B}$. thuringiensis subsp. aizawai - Bta (Xentari ${ }^{\circledR} 10 \mathrm{~g} \mathrm{~L}^{-1}$ ), e testemunha, em delineamento experimental de blocos ao acaso com quatro repetições. A dimensão da parcela foi de $4 \times 5 \mathrm{~m}$, contendo 20 plantas/linha e 80 plantas no total. Efetuaram-se três aplicações dos inseticidas, aos 15, 30 e 45 dias, após a emergência das plantas, utilizando-se pulverizador costal de 20L, com bico tipo leque e sem a adição de espalhante adesivo. Foram atribuídas notas a 10 plantas das duas fileiras centrais da área útil de cada parcela para avaliação da injúria foliar, antes de cada pulverização, segundo a escala de Carvalho (1970): 0 - plantas sem injúrias nas folhas; 1 - plantas com folhas raspadas; 2- plantas com furos nas folhas; 3 - plantas com furos nas folhas e alguma lesão no cartucho; 4 - plantas com o cartucho destruído; 5 - plantas mortas.

Em 2006, realizou-se outro experimento com o milho híbrido duplo AG 1051, utilizando-se os tratamentos com nim (Natuneem ${ }^{\circledR} 15 \mathrm{~mL} \mathrm{~L}^{-1}$; Neemseto ${ }^{\circledR}$ 10 e $\left.15 \mathrm{~mL} \mathrm{~L}^{-1}\right)$; Bta (Xentari ${ }^{\circledR} 10 \mathrm{~g} \mathrm{~L}^{-1}$ ) e testemunha, seguindo a metodologia anterior. Foram efetuadas três aplicações dos inseticidas, mas, desta vez, em menor intervalo de tempo, aos 15, 25 e 35 dias após a emergência das plantas, utilizando-se pulverizador costal de 20L com bico tipo leque e sem a adição na calda inseticida de espalhante adesivo.
Experimento em casa-de-vegetação

Plantas de milho híbrido duplo AG 1051 foram cultivadas em casa-de-vegetação, da área de fitossanidade da Universidade Federal Rural de Pernambuco (UFRPE), utilizando-se vasos com capacidade de $5 \mathrm{~kg}$, contendo solo e húmus de minhoca na proporção de 2:1, adubados com NPK (formulação 4-14-8) e calcário (10g por vaso). Foram semeadas cinco sementes por vaso, e após o desbaste, deixaram-se apenas duas plantas. Decorridos, aproximadamente, 40 dias após a emergência, as plantas, no estádio de 10 a 12 folhas, foram pulverizadas com os inseticidas à base de nim (Natuneem ${ }^{\circledR}$ e Neemseto ${ }^{\circledR} 10 \mathrm{~mL} \mathrm{~L}^{-1}$ ) e Bta (Xentari ${ }^{\circledR} 10 \mathrm{~g} \mathrm{~L}^{-1}$ ) e a testemunha apenas com água, utilizando-se pulverizador costal de 20L. Após duas horas, cada planta foi infestada com 15 lagartas de $\boldsymbol{S}$. frugiperda, com idade de 0 a $24 \mathrm{~h}$. As lagartas foram distribuídas nas folhas próximas ao cartucho, com auxílio de um pincel de pêlo fino. Em cada tratamento, usaram-se oito vasos, cada um com duas plantas. Após 10 dias, foram atribuídas notas às plantas, segundo a escala de CARVALHO (1970). Em seguida, as plantas foram cortadas, dissecadas e foi avaliado o número de lagartas sobreviventes. O experimento foi conduzido em delineamento inteiramente casualizado com quatro tratamentos e oito repetições. Os resultados foram submetidos à análise de variância e as médias comparadas pelo teste de Tukey HSD $(\mathrm{P}<0,05)$.

\section{Experimento de campo.}

Na avaliação prévia, efetuada aos 15 dias após a emergência das plantas, na safra 2005, as injúrias provocadas por $\boldsymbol{S}$. frugiperda distribuíram-se uniformemente entre os tratamentos, não apresentando diferenças estatísticas $\left(\mathrm{F}_{4,15}=0,73 ; \mathrm{P}=0,5843\right)$ (Tabela 1$)$. Aos 30 dias, Neemseto ${ }^{\circledR}$ a $10 \mathrm{~mL} \mathrm{~L}^{-1}$ foi mais efetivo com nota 0,93 , sendo o único a diferir da testemunha $\left(\mathrm{F}_{4,15}=1,92 ; \mathrm{P}=0,0159\right)$. Aos $45 \operatorname{dias}\left(\mathrm{F}_{4,15}=1,09 ; \mathrm{P}=0,3979\right)$ e, na média geral $\left(\mathrm{F}_{4,15}=0,82 ; \mathrm{P}=0,5333\right)$, os tratamentos foram semelhantes à testemunha, com variação de notas entre 1,15 a 1,63 e 1,07 a 1,58, respectivamente.

Em virtude do crescimento das plantas com produção de novas folhas, os índices de injúrias foram reduzidos ao longo das avaliações, em maior intensidade nos tratamentos com nim e Bta, e em menor na testemunha. As precipitações pluviométricas que ocorreram na área experimental durante o período de abril a junho foi de $1.052 \mathrm{~mm}$, valor muito superior à média histórica de $445 \mathrm{~mm}$ dos últimos trinta anos (ITEP, 2008). Esse fato não previsto deve ter influenciado, negativamente, nos resultados obtidos, pois segundo GASSEN (1996), as infestações de $\boldsymbol{S}$. frugiperda são favorecidas em períodos de menores índices pluviométricos e temperaturas elevadas. 
Tabela 1 - Notas atribuídas ao ataque de plantas de milho por $\boldsymbol{S}$. frugiperda, tratadas com produtos formulados de nim e Bacillus thuringiensis subsp. aizawai (Bta), em campo durante a safra 2005 e 2006, no Sítio do Araçá, Km13, em Camaragibe (Aldeia), PE.

\begin{tabular}{|c|c|c|c|c|}
\hline \multirow{3}{*}{ Tratamento } & -----------Dias & rgência das pla & ilho------------. & \multirow{3}{*}{ Média $^{2,3}$} \\
\hline & \multicolumn{3}{|c|}{ Híbrido Duplo AG 405 / Safra 2005} & \\
\hline & $15^{1}$ & 30 & 45 & \\
\hline Natuneem ${ }^{\circledR} 10 \mathrm{~mL} \mathrm{~L}^{-1}$ & $1,75 \pm 0,12 \mathrm{a}$ & $1,38 \pm 0,14 \mathrm{ab}$ & $1,15 \pm 0,08 a$ & $1,26 \pm 0,11 \mathrm{a}$ \\
\hline Neemseto ${ }^{\circledR} 5 \mathrm{~mL} \mathrm{~L}^{-1}$ & $1,83 \pm 0,13 a$ & $1,50 \pm 0,13 a$ & $1,25 \pm 0,13 a$ & $1,38 \pm 0,13 a$ \\
\hline Neemseto $^{\circledR} 10 \mathrm{~mL} \mathrm{~L}^{-1}$ & $1,90 \pm 0,14 \mathrm{a}$ & $0,93 \pm 0,10 \mathrm{~b}$ & $1,20 \pm 0,16 a$ & $1,07 \pm 0,14 \mathrm{a}$ \\
\hline Bta $10 \mathrm{~g} \mathrm{~L}^{-1}$ & $1,95 \pm 0,13 a$ & $1,10 \pm 0,07 \mathrm{ab}$ & $1,10 \pm 0,07 a$ & $1,10 \pm 0,00 \mathrm{a}$ \\
\hline \multirow[t]{3}{*}{ Testemunha } & $1,85 \pm 0,12 \mathrm{a}$ & $1,53 \pm 0,15 a$ & $1,63 \pm 0,19 a$ & $1,58 \pm 0,05 a$ \\
\hline & \multicolumn{3}{|c|}{ Híbrido Duplo AG 1051 / Safra 2006} & \\
\hline & $15^{1}$ & 25 & 35 & \\
\hline Natuneem ${ }^{\circledR} 15 \mathrm{~mL} \mathrm{~L}^{-1}$ & $1,63 \pm 0,21 \mathrm{a}$ & $2,50 \pm 0,49 a$ & $1,88 \pm 0,39 a b$ & $2,19 \pm 0,31 \mathrm{a}$ \\
\hline Neemseto ${ }^{\circledR} 15 \mathrm{~mL} \mathrm{~L}^{-1}$ & $1,55 \pm 0,36 a$ & $2,28 \pm 0,42 a$ & $0,93 \pm 0,40 \mathrm{~b}$ & $1,61 \pm 0,68 a$ \\
\hline Neemseto $^{\circledR} 10 \mathrm{~mL} \mathrm{~L}^{-1}$ & $1,80 \pm 0,41 \mathrm{a}$ & $2,70 \pm 0,35 a$ & $1,63 \pm 0,43 a b$ & $2,17 \pm 0,54 a$ \\
\hline Bta $10 \mathrm{~g} \mathrm{~L}^{-1}$ & $1,93 \pm 0,28 a$ & $1,95 \pm 0,41 \mathrm{a}$ & $0,98 \pm 0,44 b$ & $1,47 \pm 0,49 a$ \\
\hline Testemunha & $1,93 \pm 0,31 \mathrm{a}$ & $2,68 \pm 0,41 a$ & $2,38 \pm 0,26 a$ & $2,53 \pm 0,15 a$ \\
\hline
\end{tabular}

${ }^{1}$ Avaliação pré-tratamento.

${ }^{2}$ Médias ( $\pm \mathrm{EP}$ ) seguidas da mesma letra na coluna não diferem estatisticamente pelo teste de Tukey HSD (P<0,05). Valores obtidos de quatro repetições

Na safra 2006, as notas atribuídas às injúrias também variaram entre as observações (Tabela 1 ). Aos 15 dias, as notas foram uniformes em todos os tratamentos, variando de 1,55 a 1,93 $\left(\mathrm{F}_{4,15}=0,22\right.$; $\mathrm{P}=0,9233)$. Após a primeira pulverização, aos 25 dias, as notas variaram de 1,95 (Bta) a 2,68 (Testemunha), mesmo assim, não houve diferença estatística entre os tratamentos $\left(\mathrm{F}_{4,15}=0,90 ; \mathrm{P}=0,4881\right)$. Aos 35 dias, após a segunda pulverização, os melhores resultados foram obtidos com os tratamentos Neemseto $15 \mathrm{~mL} \mathrm{~L}^{-1}(0,93)$ e Bta $10 \mathrm{~g} \mathrm{~L}^{-1}(0,98)$, que foram os únicos que diferiram da testemunha $(2,38)\left(\mathrm{F}_{4,15}=5,79 ; \mathrm{P}=0,0050\right)$. Na média geral, todos os tratamentos foram semelhantes à testemunha $\left(\mathrm{F}_{415}=3,06 ; \mathrm{P}=0,0597\right)$, apesar de as notas variarem de 2,53 (Testemunha) a 1,47 (Neemseto ${ }^{\circledR} 15 \mathrm{~mL}$ $\left.\mathrm{L}^{-1}\right)$.

A patogenicidade de Bta para lagartas de $\boldsymbol{S}$. frugiperda em condições de campo, obtida nesse trabalho, é de grande importância, tendo em vista que outras formulações de Bt existentes no mercado brasileiro, como exemplo o Dipel, não são eficientes no controle dessa praga.

Experimento em casa-de-vegetação.

Os tratamentos, à base de nim e Bta, foram altamente eficientes no controle de lagartas de $\boldsymbol{S}$. frugiperda, apresentando notas 0,$7 ; 0,6$; e 0,3 , respectivamente, diferindo apenas da testemunha $(3,1)$ $\left(\mathrm{F}_{3,60}=35,01 ; \mathrm{P}<0,0001\right)$ (Tabela 2). O número de lagartas sobreviventes também foi afetado pelos tratamentos, com maior destaque para Natuneem ${ }^{\circledR} 10 \mathrm{~mL} \mathrm{~L}^{-1}$, que reduziu em $100 \%$ a sobrevivência, seguido por Neemseto ${ }^{\circledR} 10 \mathrm{~mL} \mathrm{~L}^{-1}$, Bta $10 \mathrm{~g} \mathrm{~L}^{-1}$ e testemunha, com médias de 0,3;0,3 e 3,0 lagartas sobreviventes, respectivamente $\left(\mathrm{F}_{3,60}=24,35 ; \mathrm{P}=0,0001\right)$.

Lagartas mais novas de $\boldsymbol{S}$. frugiperda foram mais suscetíveis aos produtos de nim. A morte dos insetos-alvo, provocada pelo mim, depende da concentração e do tempo de exposição (SCHMUTTERER, 1988). Outro aspecto a considerar é que os insetos são capazes de diferenciar as partes tratadas e não tratadas (SCHMUTTERER, 1990), evidenciando que a pulverização deve ser cuidadosamente realizada em alto volume e uma uniforme distribuição da calda.

No presente estudo, os inseticidas à base nim e Bta mostraram-se promissores para o controle de S. frugiperda em casa-de-vegetação e em campo. No entanto, sugere-se que outros experimentos sejam realizados em campo, testando-se novos intervalos de aplicação sob uma maior pressão populacional da praga, visando a confirmar a eficácia desses produtos, para poder utilizá-los como uma alternativa aos inseticidas sintéticos, em programas de manejo integrado de pragas.

\section{AGRADECIMENTOS}

Ao Conselho Nacional de Desenvolvimento Científico e Tecnológico (CNPq), pela concessão da bolsa ao 
Tabela 2 - Notas atribuídas e lagartas sobreviventes (média \pm EP) de $\boldsymbol{S}$. frugiperda em plantas de milho Híbrido Duplo AG 1051, tratadas com produtos formulados de nim e Bacillus thuringiensis subsp. aizawai (Bta) em casa-de-vegetação.

\begin{tabular}{lcc}
\hline Tratamento & Escala de notas $^{1,2}$ & Lagartas sobreviventes $^{2,3}$ \\
\hline Natuneem $^{\circledR} 10 \mathrm{~mL} \mathrm{~L}^{-1}$ & $0,7 \pm 0,23 \mathrm{~b}$ & $-{ }^{-}$ \\
Neemseto $^{\circledast} 10 \mathrm{~mL} \mathrm{~L}^{-1}$ & $0,6 \pm 0,22 \mathrm{~b}$ & $0,3 \pm 0,16 \mathrm{~b}$ \\
Bta $10 \mathrm{~g} \mathrm{~L}^{-1}$ & $0,3 \pm 0,22 \mathrm{~b}$ & $0,3 \pm 0,25 \mathrm{~b}$ \\
Testemunha & $3,1 \pm 0,09 \mathrm{a}$ & $3,0 \pm 0,57 \mathrm{a}$ \\
\hline
\end{tabular}

${ }^{1}$ Escala de notas: 0 - plantas sem injúrias nas folhas; 1 - plantas com folhas raspadas; 2 - plantas com furos nas folhas; 3 - plantas com furos nas folhas e alguma lesão no cartucho; 4 - plantas com o cartucho destruído; 5 - plantas mortas (Carvalho 1970).

${ }^{2}$ Médias (quatro repetições) seguidas de mesma letra na coluna não diferem entre si pelo teste de Tukey HSD $(\mathrm{P}<0,05)$.

${ }^{4}$ Ausência de sobrevivência (Exclusão da análise estatística).

primeiro autor, possibilitando a realização deste trabalho. Ao Prof. Jorge Braz Torres, pelas valiosas sugestões.

\section{REFERÊNCIAS}

CARVALHO, R.P.L. Danos, flutuação da população, controle e comportamento de Spodoptera frugiperda (J.E. Smith, 1797) e susceptibilidade de diferentes genótipos de milho, em condições de campo. 1970. 170f. Tese (Doutorado em Entomologia) - Escola Superior de Agricultura "Luiz de Queiroz”/USP.

CRUZ, I. A lagarta do cartucho do milho. Sete Lagoas: EMBRAPA - CNPMS, 1995. 45p. (EMBRAPA - CNPMS. Circular Técnica, n.21)

GASSEN, D.N. Manejo de pragas associadas à cultura do milho. Passo Fundo: Aldeia Norte, 1996.

134p.

ITEP (INSTITUTO TECNOLÓGICO DO ESTADO DE PERNAMBUCO). 2008. Disponível na Internet: <http:// www.itep.br/meteorologia/lamepe/index.html>. On line. Acesso em: 01 mar 2008.

MARTINEZ, S.S.; VAN EMDEN, H.F. Growth disruption, abnormalities and mortality of Spodoptera littoralis (Boisduval) (Lepidoptera: Noctuidae) caused by azadirachtin. Neotropical Entomology, Londrina, v.30, n.1, p.113-125, 2001.
MARTINEZ, S.S. (ed.). O nim - Azadirachta indica: Natureza, usos múltiplos, produção. Londrina: Instituto Agronômico do Paraná, 2002. 142p.

SCHMUTTERER, H. Potential of azadirachtin-containing pesticides for integrated pest control in developing and industrialized countries. Journal of Insect Physiology, Oxford, v.34, n.7, p.713-719, 1988. Disponível em: <http:// www.sciencedirect.com/science?_ob=ArticleURL\&_udi=B6T3F49N9RMM-2JD\&_user $=687358$ \&_rdoc $=1 \&$ fmt $=$ \&_orig $=$ se arch \&_sort $=$ d \& vi e w $=$ c \&_a c c t $=$ C 0000378 $99 \&$ \& version $=1 \&$ _ url Version $=0 \&$ _ u se rid $=687358 \& \mathrm{md} 5=9 \mathrm{e} 7 \mathrm{cb} 4 \mathrm{e} 500457 \mathrm{ef7aa} 1047534053$ c533.> Doi: 10.1016/0022-1910(88)90082-0.

SCHMUTTERER, H. Properties and potential of natural pesticides from the neem tree, Azadirachta indica. Annual Review of Entomology, Palo Alto, v.35, p.271-297, 1990. Disponível em: <http://arjournals.annualreviews.org/doi/abs/ 10.1146\%2Fannurev.en.35.010190.001415>. Doi: 10.1146/ annurev.en.35.010190.001415.

SIEGEL, J.P. The mammalian safety of Bacillus thuringiensisbased insecticides. Journal of Invertebrate Pathology, v.77, p.13-21, 2001. Disponível em: < The mammalian safety of Bacillus thuringiensis-based insecticides>. Doi: 10.1006/ jipa.2000.5000. 